\title{
Hepatitis B virus X protein promotes the growth of hepatocellular carcinoma by modulation of the Notch signaling pathway
}

\author{
FAN WANG ${ }^{*}$, HAIYAN ZHOU* $^{*}$ YUZHEN YANG, XIUMEI XIA, QIAN SUN, JIN LUO and BIN CHENG \\ Department of Gastroenterology and Hepatology, Tongji Hospital, Tongji Medical College, \\ Huazhong University of Science and Technology, Wuhan 430030, P.R. China
}

Received October 8, 2011; Accepted December 12, 2011

DOI: $10.3892 /$ or.2012.1620

\begin{abstract}
Hepatitis B virus X protein ( $\mathrm{HBx}$ ) plays a crucial role in the development of hepatocellular carcinoma (HCC), however, little is known about the mechanism. Here, we investigated the relationship between $\mathrm{HBx}$ and Notch signaling in HepG2 cells after they were transfected with the HBx gene. It was found that HBx upregulated the expression of Notch-1, Jagged-1 and Hes-1 at the transcriptional level by binding to the Notch-1 intracellular domain, which is congruent with the observations of enhanced malignant biological activities of HBx-transfected HepG2 cells compared with normal HepG2 cells. However, while Notch signaling was blocked, the HBx-induced abnormalities were partially reversed. These findings suggest that $\mathrm{HBx}$ may promote the progression of $\mathrm{HCC}$ via the activated Notch pathway.
\end{abstract}

\section{Introduction}

Hepatocellular carcinoma (HCC) is one of the most malignant tumors worldwide and Hepatitis B virus (HBV) has been identified as the most important risk factor for developing HCC (1-3). Of the four proteins translated by HBV, the X-gene product (HBx) has been most closely associated with the HCC pathogenesis (4). The correlation between $\mathrm{HBx}$ and HCC development has been extensively studied and the oncogenic roles of $\mathrm{HBx}$ include the following: activation of a variety

Correspondence to: Professor Bin Cheng, Department of Gastroenterology and Hepatology, Tongji Hospital, Tongji Medical College, Huazhong University of Science and Technology, 1095 Jiefang Street, Wuhan 430030, P.R. China

E-mail: b.cheng@tjh.tjmu.edu.cn

*Contributed equally

Abbreviations: HCC, hepatocellular carcinoma; HBV, hepatitis B virus; HBx, HBV X protein; Notch-IC or NICD, Notch intracellular domain; CSL, mammalian $\mathrm{CBF} 1$, Drosophila $\mathrm{Su}(\mathrm{H})$, C. elegans LAG-1; DAPT, $\gamma$-secretase inhibitor N-[N-(3,5-difluorophenacetyl)L-alanyl]-S-phenylglycine t-butyl ester; qRT-PCR, quantitative real-time RT-PCR

Key words: HBx, HepG2, Notch, NICD, DAPT of transcription factors such as nuclear factor- $\kappa \mathrm{B}(\mathrm{NF}-\kappa \mathrm{B})$ (5), activator protein 1 (AP-1) (6), cAMP-responsive element binding protein/activating transcription factor 2 (CREB/ ATF-2) (7); interaction with cellular oncogenes, such as Ras (8) and Src (9); regulation of cell apoptosis and the cell cycle by interacting with caspases, CDK, CKI, and survivin (10,11); and stimulation of cell signaling pathways, such as the Wnt (12), the Ras/MAPK (13), and the PI3K-Akt/PKB pathway (14).

Notch signaling is a highly evolutionarily conserved pathway which plays a pivotal role in regulating the development of organs and tissues by affecting cell proliferation, differentiation, apoptosis and stem cell maintenance (15). In mammals, the Notch family consists of four transmembrane receptors (Notch-1-Notch-4) and five ligands (Jagged-1, Jagged-2, Dll-1, Dll-3 and Dll-4) (16). Upon receptor-ligand binding, the Notch receptor is proteolytically cleaved by a $\gamma$-secretase complex (17), which results in releasing of the Notch intracellular domain (NICD) (18). Then NICD translocates into the nucleus and bands to the transcriptional factors known as CSL (CBF-1/suppressor of hairless/ Lag-1), leading to the transcriptional activation of Notch target genes, including basic helix-loop-helix (bHLH) proteins such as HES-1 and HES-5 (19,20). Mounting evidence shows that perturbation of Notch signaling often leads to tumorigenesis (21-24). Some studies have shown the potential roles of Notch signaling in the development of $\operatorname{HCC}(25,26)$.

However, up to now, only a few reports about the role of Notch signaling in HBx-related HCC. In this study, we investigated the relationship of the Notch pathway with $\mathrm{HBx}$ in HepG2 cells, and found that HBx can enhance the progression of HCC via the activation of Notch pathway, which may provide some new clues for the potential role of Notch signaling in $\mathrm{HBx}$-associated liver cancer.

\section{Materials and methods}

Cell culture. The human hepatoma cell line HepG2 were obtained from the American Type Culture Collection (ATCC; Manassas, VA, USA). The HepG2/HBx and HepG2/pcDNA3.1 cell lines, were derived from HepG2 cells by transfecting with HBx expression plasmid or an empty plasmid (pcDNA3.1(+)/ V5-HisB), respectively. Both cell lines have been successfully established (27). All cell lines were cultured in DMEM (Gibco, Carlsbad, CA, USA) supplemented with $10 \%$ fetal bovine 
serum (FBS; Gibco, Grand Island, NY, USA) and maintained in humidified incubator at $37^{\circ} \mathrm{C}$ in a $5 \% \mathrm{CO}_{2}$ atmosphere.

DAPT treatment. The $\gamma$-secretase inhibitor $\mathrm{N}-[\mathrm{N}-(3,5-$ difluorophenacetyl)-L-alanyl]-S-phenylglycine t-butyl ester (DAPT) was purchased from the Sigma-Aldrich Company (St. Louis, MO, USA). DAPT was dissolved in 100\% dimethylsulphoxide (DMSO, Sigma) to make a stock solution of $10 \mathrm{mM}$, which was then diluted in culture medium to obtain the desired concentrations of $1,5,10$ and $20 \mu \mathrm{M}$. DMSO diluted in culture medium at the final concentration of $0.05 \%$ without DAPT was designated as $0 \mu \mathrm{M}$. Untreated cells were those incubated in the culture medium without any additives. Cells treated with or without DAPT were cultured for $48 \mathrm{~h}$, after which the total RNA or protein was extracted and flow cytometry was carried out.

Cell proliferation and viability assays. Cell proliferation assays were performed by using a Cell Counting Kit-8 (Dojindo, Kumamoto, Japan) according to the manufacturer's instructions. Briefly, 1x10 $0^{4}$ cells/well were plated in 96-well plates and cultured with growth medium. At the indicated time points, the medium was aspirated. Then $100 \mu \mathrm{l}$ serum-free DMEM and $10 \mu \mathrm{l}$ WST-8 [2-(2-methoxy-4-nitrophenyl)3-(4-nitrophenyl)-5-(2,4-disulfophenyl)-2H tetrazolium, monosodium salt] were added to each well followed by incubation at $37^{\circ} \mathrm{C}$ for $1.5 \mathrm{~h}$. Absorbance was measured at $450 \mathrm{~nm}$ with a reference wavelength of $630 \mathrm{~nm}$ on a spectrophotometer (Molecular Devices, Sunnyvale, CA). To evaluate the viability of HepG2/HBx cells, $1 \times 10^{4}$ cells/well were plated in 96-well plates followed $12 \mathrm{~h}$ later by the addition of DAPT at concentrations of $0,1,5,10$ or $20 \mu \mathrm{M}$. The cell viability was assessed as the percent of viable cells relative to the untreated control cells, which was determined for each concentration using the following equation: $\%$ viability $=\mathrm{OD}_{\text {experiment }} / \mathrm{OD}_{\text {control }} \times 100 \%$. Control cells were considered as $100 \%$ viable. All experiments were repeated five times.

Cell cycle and apoptosis analysis by flow cytometry. After treatment with or without DAPT for $48 \mathrm{~h}$, cells were harvested, immediately fixed in $75 \%$ ethanol at $4^{\circ} \mathrm{C}$ overnight, treated with $50 \mathrm{mg} / 1 \mathrm{RNAse}$ A (Sigma) for $30 \mathrm{~min}$ at $37^{\circ} \mathrm{C}$, and stained with $50 \mathrm{mg} / \mathrm{l}$ PI (Sigma) for $10 \mathrm{~min}$. Samples were then analyzed for their DNA content by a FACSAria Cell Cytometer (BD Biosciences, San Jose, CA, USA). The data were analyzed with the CellQuest software (BD Biosciences). Apoptosis analysis was performed by using a Annexin-V-FITC kit (Bender MedSystems, Burlingame, CA, USA) according to the manufacturer's instructions. The percentage of cells that were Annexin-V positive but PI negative was compared among the different treatment groups.

Immunofluorescence assays. Immunofluorescence assays were performed as previously described (28). HepG2/HBx cells were cultured on glass coverslips for $24 \mathrm{~h}$ and fixed with $4 \%$ paraformaldehyde. The fixed cells were incubated with anti-HBx (1:200, Santa Cruz Biotechnology, Santa Cruz, CA) and anti-NICD (1:200, Santa Cruz Biotechnology) for $12 \mathrm{~h}$ at $4^{\circ} \mathrm{C}$, then incubated with $\mathrm{Cy} 3$-conjugated goat antimouse IgG (1:100, Boster, China) and FITC-conjugated goat anti-rabbit $\operatorname{IgG}(1: 100$, Boster) for $1 \mathrm{~h}$. Nuclei were stained with diamidinophenyl indole (DAPI) (Boster). The stained cells were observed using a Fluoview FV1000 laser scanning confocal microscope (Olympus, Japan).

Co-immunoprecipitation assays. Co-immunoprecipitation assays were performed as previously described (28). HepG2/ $\mathrm{HBx}$ cells were lysed, and the lysates were pretreated with protein G-agarose (Santa Cruz Biotechnology) to remove nonspecifically bound proteins. After centrifugation, one third of the supernatants were stored at $-80^{\circ} \mathrm{C}$ as a positive control. The remaining supernatant samples were incubated for $2 \mathrm{~h}$ at $4^{\circ} \mathrm{C}$ with $1 \mu \mathrm{g}$ of non-immune mouse IgG or mouse anti-HBx (Santa Cruz Biotechnology), for the negative control and the experimental group, respectively. Then, the mixture was incubated for $1 \mathrm{~h}$ to overnight at $4^{\circ} \mathrm{C}$ with $20 \mu \mathrm{l}$ protein G-agarose (Santa Cruz Biotechnology). The immunocomplexes were extensively washed with PBS, samples were boiled in electrophoresis sample buffer and assayed by Western blot analysis.

Real-time PCR analysis. Total RNA was isolated from cultured cells using TRIzol reagent (Invitrogen, Carlsbad, CA, USA) and cDNA was synthesized from $2 \mu \mathrm{g}$ of total RNA using Moloney murine leukemia virus reverse transcriptase (MMLV) (Promega, Madison, WI, USA). Real-time quantitative PCR (qRT-PCR) using SYBR Premix (DRR041A, Takara, Japan) was performed as previously described (29). Amplifications were performed in a LightCycler machine (Roche Diagnostics, Basel, Switzerland) following the manufacturer's instructions. The HepG2 cDNA was used as standard cDNA. A standard curve for each gene was generated from serially diluted standards, and values for unknown samples were extrapolated. $\beta$-actin was used as an internal control to normalize samples. All standards and samples were assayed in triplicate. The primer sequences used to amplify specific target genes are listed in Table I.

Western blot analysis. Cells were lysed as previously described (29) and the lysates were subjected to electrophoresis on SDS-PAGE and transferred to PVDF membranes (Millipore, Billerica, MA). The blotted membranes were blocked and subsequently incubated with rabbit anti-Jagged-1 (1:600), rabbit anti-Notch-1 (1:1,000), rabbit anti-Notch-1-IC (1:1,000), rabbit anti-Hes-1 (1:600), and rabbit anti-actin (1:1,000). All antibodies were from Santa Cruz Biotechnology except for anti-Notch-1-IC which was obtained from Cell Signaling Technology (Danvers, MA). After incubation with horseradish peroxidase-labeled secondary antibody (1:5,000-10,000; Santa Cruz Biotechnology), visualization was performed by an enhanced chemiluminescence kit (Pierce, Rockford, IL) and exposure to X-ray film (Kodak, Rochester, NY). Immunoblotting with the anti-actin antibody was used as an internal control to confirm equivalent protein loading. Each experiment was performed at least 3 times. The relative intensity of each protein band was scanned by Quantity One software (Bio-Rad Laboratories, Hercules, CA, USA).

Statistics. SPSS version 17.0 software (SPSS for Windows, Inc., Chicago, IL, USA) was used for all statistical analyses. All results are expressed as mean \pm SEM. Statistical analysis of 
Table I. Primer sequences for real-time polymerase chain reaction analysis.

\begin{tabular}{llcc}
\hline Gene & \multicolumn{1}{c}{ Primer sequence } & PCR product size (bp) & GenBank accession no. \\
\hline Jagged-1 & F: 5'-CAACACGGTCCCCATCAAG-3' & & NM_000214.2 \\
& R: 5'-TACTTCAGAATTGTGTGTCCTTATTTTAGA-3' & 109 & NM_017617.3 \\
Notch-1 & F: 5'-CCGCAGTTGTGCTCCTGAA-3' & & NM_005524.2 \\
& R: 5'-ACCTTGGCGGTCTCGTAGCT-3' & 122 & Nes-1 \\
\cline { 2 - 2 } & F: 5'-GCTAAGGTGTTTGGAGGCT-3' & & NM_001101.3 \\
& R: 5'-CCGCTGTTGCTGGTGTA-3' & 157 & F: 5'-GTTGCGTTACACCCTTTCTTG-3'
\end{tabular}

F, forward; R, reverse.

$\mathbf{A}$

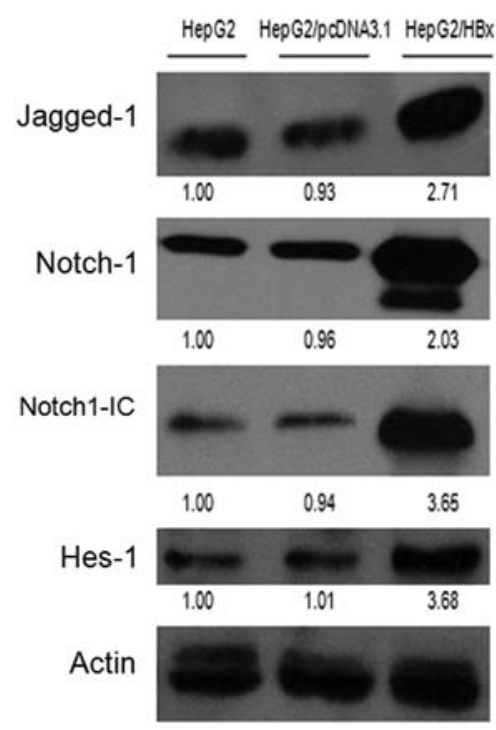

C

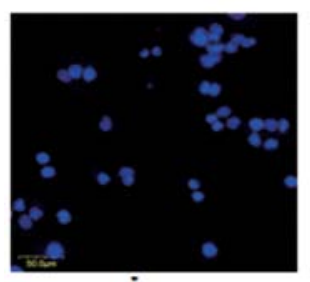

i

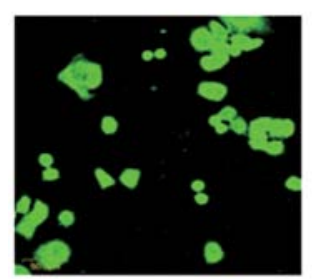

iii

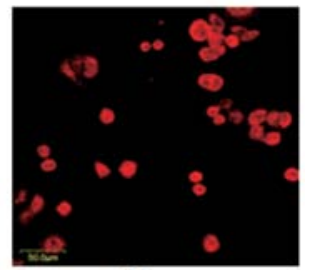

ii

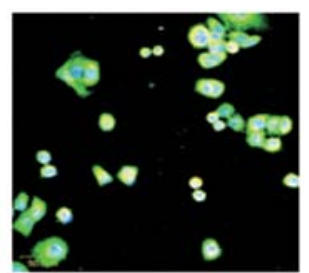

iv

D

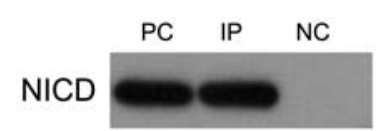

Jagged-1

B

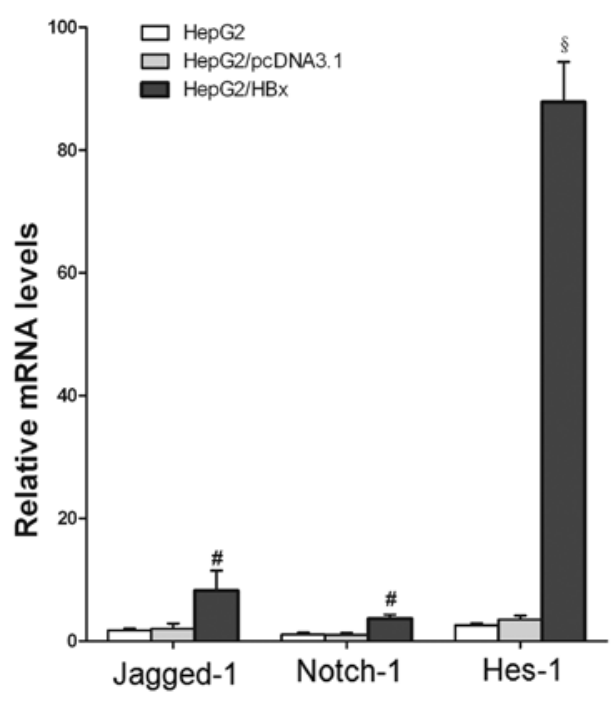

Figure 1. Expression of Notch signaling pathway components and the physical interaction of NICD with HBx in HBx-expressing cells. (A) The protein levels of Notch signaling pathway components were detected using western blot analysis. Actin was used as an internal control for equal loading of samples. Representative blots shown were from three independent experiments with identical results. The relative ratios of each band were normalized to actin and are shown below each western blot band. (B) Jagged-1, Notch-1, Hes-1 mRNA expression levels were determined by quantitative real-time RT-PCR. Results are mean \pm SEM of 3 independent experiments. ${ }^{~} \mathrm{P}<0.01$ and ${ }^{\S} \mathrm{P}<0.001$ vs. control (HepG2 cells). (C) Confocal microscopy imaging of the co-localization of NICD with HBx in L02/HBx cells. The nuclei were stained with DAPI (blue) (i), HBx protein was stained with Cy3-conjugated goat anti-mouse IgG (red) (ii) and the protein of NICD was stained with FITC-conjugated goat anti-rabbit IgG (green) (iii). Yellow staining indicates overlapping areas of red and green fluorescent labels (iv). (D) Co-immunoprecipitation assay of the interaction of NICD and Jagged-1 with $\mathrm{HBx}$ in HepG2/HBx cells. The preparation of proteins was described in Materials and methods. All the proteins were analyzed by western blot analysis using anti-NICD, anti-Jagged-1. The data shown are representative of 3 independent experiments. PC, positive control; IP, co-immunoprecipitation of proteins treated with anti-HBx; $\mathrm{NC}$, co-immunoprecipitation of proteins treated with non-immune mouse $\mathrm{IgG}$ (negative control). 
A

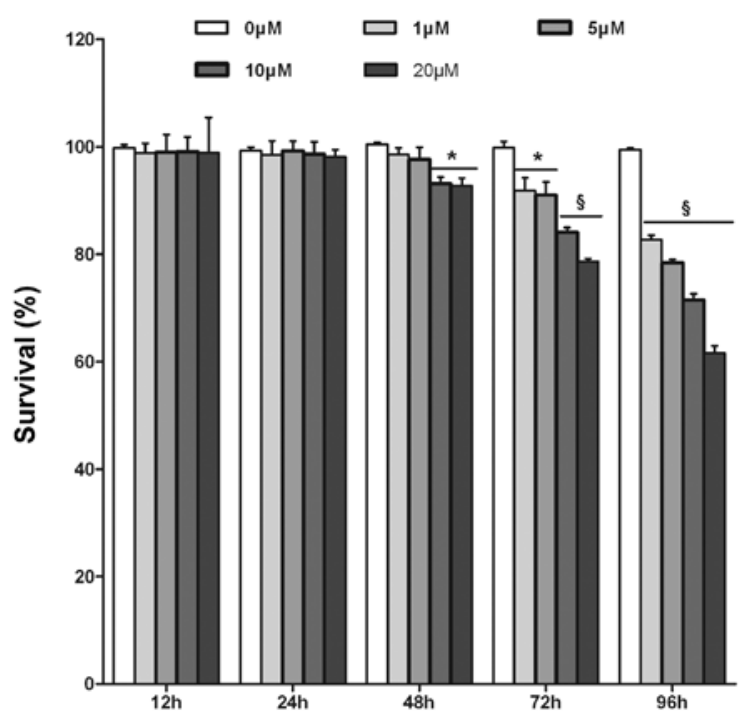

C

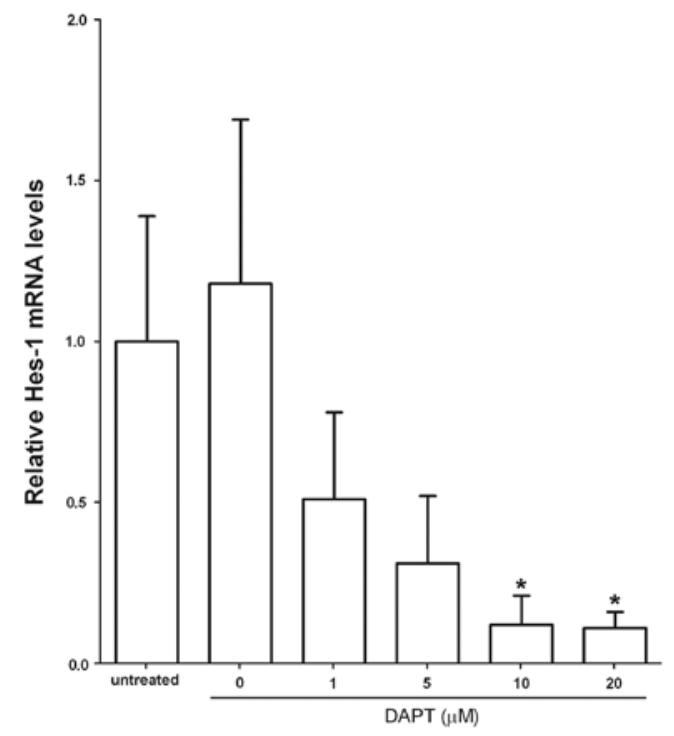

the data was performed using a standard one-way ANOVA or one-way ANOVA for repeated measures, followed by the least significant difference (LSD) post-hoc test. Bonferroni's correction was used to adjust for multiple comparisons. A 2-tailed Student's paired t-test was also used to compare the differences in the values between two groups. A P-value $<0.05$ was considered to be statistically significant.

\section{Results}

The Notch signaling pathway is activated in HepG2/HBx cells. Previously, we observed that the HBx protein stimulated the proliferation as well as the cell cycle, inhibited the apoptosis of HepG2 cells significantly, and promoted tumour growth in nude mice (27). To investigate the potential involvement of Notch signaling in the development of $\mathrm{HCC}$, we performed western blot analyses in HepG2, HepG2/pcDNA3.1 and HepG2/HBx cells. As shown in Fig. 1A, the protein levels of Jagged-1, Notch-1, Notch-1C and Hes-1 in HepG2/HBx cells were elevated relative to the controls. Then, we analyzed the mRNA levels of Jagged-1, Notch-1 and Hes-1 using quantitative real-time RT-PCR (qRT-PCR) and found that their mRNA

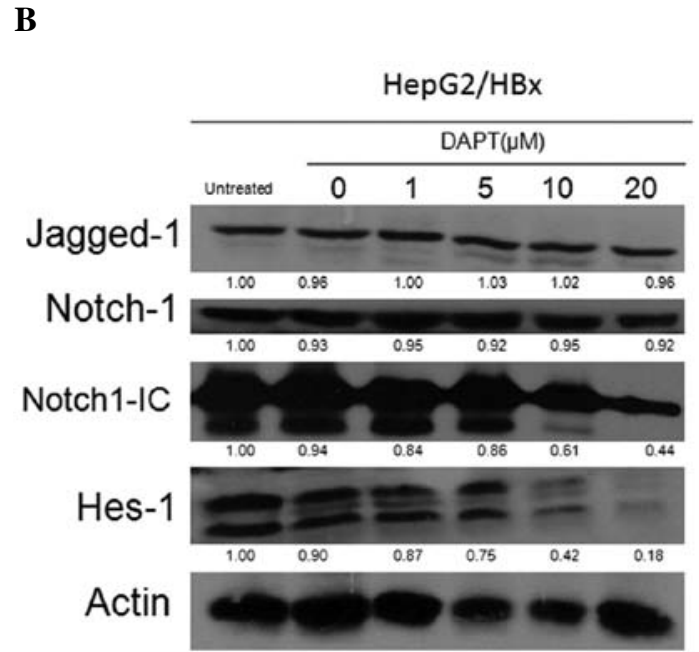

Figure 2. (A) Effects of inhibition of Notch signaling on the growth of HepG2/HBx cells. The effect of DAPT on cell viability of HepG2/HBx cells was determined by the WST- 8 assay as described in Materials and methods. Results are expressed as mean \pm SEM of five samples. ${ }^{*} \mathrm{P}<0.05$ vs. control (non-DAPT); ${ }^{\circledR} \mathrm{P}<0.001$ vs. control. (B and C) Notch signaling in HepG $2 / \mathrm{HBx}$ cells by the $\gamma$-secretase inhibitor DAPT. (B) Inhibition of Notch signaling in HepG2/HBx cells with indicated concentrations of DAPT was assessed by immunoblotting. Cells were harvested after $48 \mathrm{~h}$ of treatment. Actin was used as an internal control for equal loading of samples. Representative blots shown are from three independent experiments with identical results. The relative ratios of each band were normalized to actin and are shown below each western blot band. (C) Hes-1 mRNA levels were assessed by qRT-PCR in DAPT-treated HepG2/HBx cells. Cells were harvested after $48 \mathrm{~h}$ of treatment. Data shown are mean \pm SEM of representative experiment performed in triplicate. ${ }^{*} \mathrm{P}<0.01$ vs. control (untreated cells).

levels were significantly increased in $\mathrm{HepG} 2 / \mathrm{HBx}$ cells as compared with the controls (Fig. 1B).

Notch-1 colocalizes and interacts with HBx in HepG2/HBx cells. To study the possible relationship between Notch-1 and $\mathrm{HBx}$, we performed immunofluorescence and co-immunoprecipitation assays. As shown in Fig. 1C, the nuclei were stained blue (i), HBx was stained red (ii) whereas the NICD was stained green (iii) in HepG2/HBx cells. Yellow staining in the dual-labeling experiments indicates overlapping areas of red and green fluorescent labels (iv), suggesting co-localization of NICD with HBx in the HepG2/HBx cells. The compounds immunoprecipitated with anti-HBx or non-immune mouse IgG from HepG2/HBx cells were subjected to western blot analysis with anti-NICD and anti-Jagged-1, respectively. As shown in Fig. 1D, NICD was co-immunoprecipitated with HBx. No specific interaction was found between Jagged-1 and $\mathrm{HBx}$ or the protein immunoprecipitated by non-immune $\operatorname{IgG}$, indicating the specificity of the NICD-HBx interaction.

Inhibition of Notch signaling attenuates the growth of Hep G2/ $H B x$ cells. In order to further establish that active Notch 

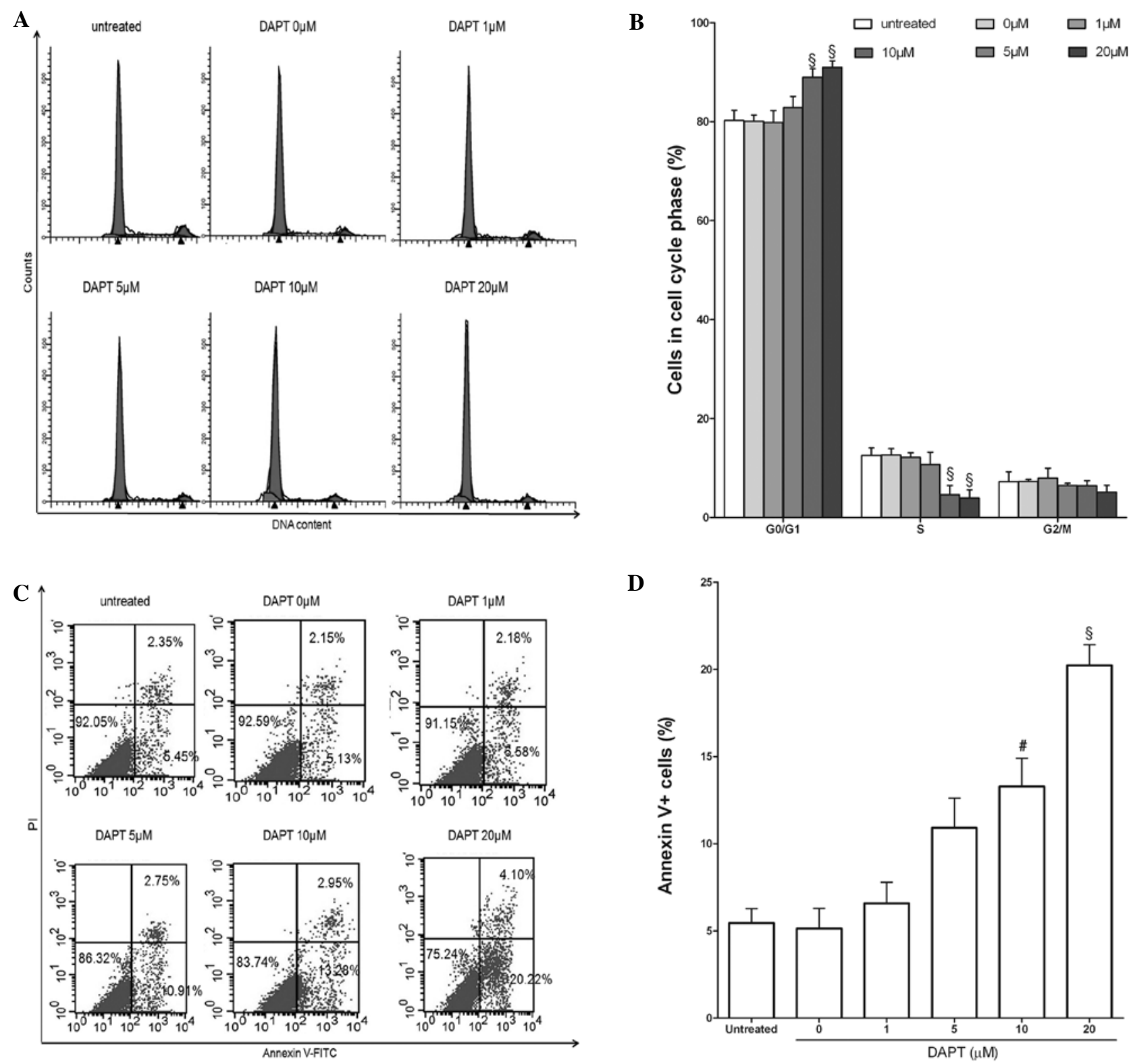

Figure 3. Effects of the inhibition of Notch signaling in G0/G1 distribution, and apoptosis in HepG2/HBx cells. (A and B) The cell-cycle distribution of HepG2/ HBx cells was examined by PI staining and flow cytometry synchronized by serum starvation, and treated with or without DAPT for $48 \mathrm{~h}$. Results shown are from a representative experiment (A) or mean \pm SEM of 3 experiments (B), each performed in triplicate. ${ }^{\S} \mathrm{P}<0.05$ vs. control (untreated cells). (C and D) Apoptosis of HepG2/HBx cells treated with or without DAPT for $48 \mathrm{~h}$ detected by Annexin-V and PI staining using FACS analysis. The percentage of cells that were Annexin- $V$ positive, but PI negative were compared among different groups. Results shown are from a representative experiment (C) or mean \pm SEM of 3 experiments (D), each performed in triplicate. ${ }^{\#} \mathrm{P}<0.01$ and ${ }^{\S} \mathrm{P}<0.001$ vs. control (untreated cells).

signaling is important for HBx to function as an oncoprotein, we treated HepG2/HBx cells with various concentrations of DAPT for 12 to $96 \mathrm{~h}$ and assessed cell proliferation by the WST-8 assay. As shown in Fig. 2A, in HepG2/HBx cells, increasing concentrations and durations of treatment with DAPT resulted in a decrease of cell viability. In contrast, treatment with various concentrations of DAPT for 12 or $24 \mathrm{~h}$ did not produce any significant reduction in cell viability. However, treatment of DAPT for $>48 \mathrm{~h}$ resulted in significant dose- and time-dependent reduction in cell viability of HepG2/ HBx cells. A significant reduction in cell viability by DAPT treatment was observed at concentrations of 10 and $20 \mu \mathrm{M}$ after $48 \mathrm{~h}$, with inhibition rates of 7.30 and $7.73 \%$, respectively $(\mathrm{P}<0.05$, Fig. 2A). We, therefore, selected the DAPT treatment time point of $48 \mathrm{~h}$ for further studies.
Confirmation of the inhibition of Notch signaling in HepG2/ HBx cells. HepG2/HBx cells treated with various concentrations of DAPT for $48 \mathrm{~h}$ and assessed for the DAPT inhibitory effects on Notch-1 signaling by western blot analysis of Jagged-1, Notch-1, Notch-1-IC, Hes-1 protein levels, and by qRT-PCR of Hes-1 transcripts. As shown in Fig. 2B and C, DAPT treatment greatly reduced the amount of Notch-1-IC and Hes-1 protein in a dose-dependent manner, while there was no significant effect on Jagged-1 and Notch-1 levels (Fig. 2B). A significant reduction of Notch-1-IC and Hes-1 protein levels were observed at 10 and $20 \mu \mathrm{M}$ of DAPT treatment. qRT-PCR experiments showed that the downregulation of Hes-1 transcripts in HepG2/HBx cells occurred after treatment with DAPT at $10(\mathrm{P}<0.05)$ and $20 \mu \mathrm{M}(\mathrm{P}<0.05)$ (Fig. 2C). These data were consistent with the previous results that treatment 
with 10 and $20 \mu \mathrm{M}$ DAPT for $48 \mathrm{~h}$ significantly inhibited the proliferation of HepG2/HBx cells.

Inhibition of Notch signaling arrests G0/G1 phase, shortens the $S$ phase and induces apoptosis in HepG2/HBx cells. We performed PI staining and flow cytometry to define the cell-cycle distribution of DAPT-treated HepG2/HBx cells. Treatment of HepG2/HBx cells with $0-20 \mathrm{M}$ of DAPT for $48 \mathrm{~h}$ resulted in arrest in the G0/G1 phase and shortening of the $\mathrm{S}$ phase. The proportion of HepG2/HBx cells in the G0/G1 phase was significantly increased at concentrations of $10(88.99 \%$, $\mathrm{P}<0.05)$ and $20 \mu \mathrm{M}(90.95 \%, \mathrm{P}<0.05)$, compared to that of $80.23 \%$ in untreated control cells (Fig. 3A and B). The proportion of cells in $\mathrm{S}$ phase was significantly decreased at concentrations of $10(4.60 \%, \mathrm{P}<0.05)$ and $20 \mu \mathrm{M}(3.93 \%, \mathrm{P}<0.05)$, relative to that of $12.55 \%$ in untreated control cells (Fig. 3A and B). No significant difference was observed between the $\mathrm{G} 2 / \mathrm{M}$ phases. As shown in Fig. 3C and D, the apoptotic rates were increased from $5.45 \%$ in non-DAPT-treated cells to $13.28 \%$ at a concentration of $10 \mu \mathrm{M}(\mathrm{P}<0.01$, Fig. 3C and D) and to $(20.22 \%)$ at $20 \mu \mathrm{M}(\mathrm{P}<0.001$, Fig. 3C and D) after treatment with DAPT for $48 \mathrm{~h}$. Taken together, these results indicate that the inhibition of Notch signaling was associated with decreased DNA synthesis (S phase) and increased apoptosis, which contributed to the impaired growth of HepG2/HBx cells treated with DAPT.

HepG2 cells are not significantly affected by Notch inhibition. As a control, Notch signaling was blocked in HepG2 cells using DAPT as well. HepG2 cells were treated with DAPT at the final concentration of $10 \mu \mathrm{M}$ or with $0.05 \%$ DMSO. The proliferation rate of HepG2 cells was measured after DAPT treatment using WST-8 assay. No effect on cell proliferation could be observed in HepG2 cells upon ablation of Notch signaling (Fig. 4A). The protein expression level of Hes-1 was monitored using western blot analysis. As shown in Fig. 4B, treatment of HepG2 cells with DAPT led to a distinct Hes-1 downregulation after $48 \mathrm{~h}$. These results demonstrated that Notch signaling was much less important for the growth of HepG2 cells than for that of HepG2/HBx cells.

\section{Discussion}

We previously reported that $\mathrm{HBx}$ protein stimulated the proliferation as well as cell cycle, inhibited the apoptosis of HepG 2 cells significantly, and promoted tumor growth in nude mice (27). However, little is known about the mechanism of $\mathrm{HBx}$ action. A large number of studies have demonstrated that Notch signaling plays a crucial role in various malignant tumors (21-24). Expression and localization of Notch receptors and their ligands have been observed in the normal human liver tissue and the deregulated Notch signaling had been found in malignant liver tumors $(25,26)$.

To examine whether Notch signaling was involved in the HBx-related HCC, we investigated the relationship between HBx and Notch signaling in HepG2 cells after being transfected with the HBx gene. It was found that HBx protein upregulated the expression of Notch-1, Jagged-1 and Hes-1 at the transcriptional level, which is related with the stimulated growth of HepG2 cells by HBx in vitro and in vivo. However, when Notch signaling was blocked with a $\gamma$-secretase inhibitor DAPT, the
A

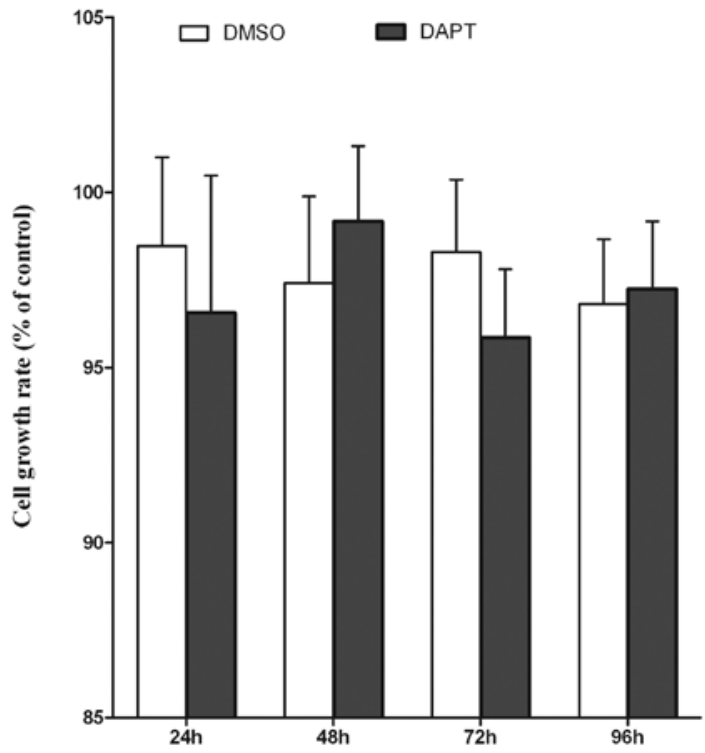

$\mathbf{B}$

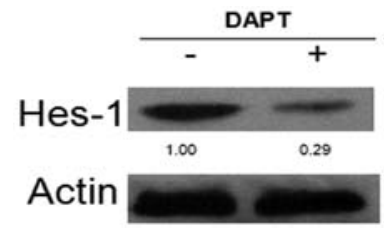

Figure 4. Inhibition of Notch signaling in HepG2 cells. HepG2 cells were treated with DAPT at the final concentration of $10 \mu \mathrm{M}$ or with $0.05 \%$ DMSO. (A) HepG2 cells $\left(1 \times 10^{4}\right)$ were cultured in medium contained DAPT or DMSO for indicated times. Cell growth was assessed by the WST-8 assay as described in Materials and methods and plotted as a percentage of that of the control (untreated cells). Bars represent the means \pm SEM of each time point from five samples. (B) HepG2 cells were harvested after $48 \mathrm{~h}$ of treatment with DAPT $(10 \mu \mathrm{M})$ or vehicle control $(0.05 \%$ DMSO). Inhibition of Notch signaling in HepG2 cells was monitored by immunoblotting for Hes-1 protein levels (+, DAPT; -, vehicle control).

inhibited Notch signaling decreased the proliferation, arrested the G0/G1 phase and shortened the $\mathrm{S}$ phase of the cell cycle and induced apoptosis in HepG2/HBx cells. It seemed that when Notch signaling was blocked, the abnormality caused by $\mathrm{HBx}$ in HepG2 cells was partially reversed. However, the inhibition of Notch signaling in untransfected HepG 2 cells had barely any effect on their growth. Moreover, we found that HBx colocalized and interacted with NICD (Notch intracellular domain) in HepG2/HBx cells. These findings demonstrated that HBx can activate Notch signaling by binding to NICD, which may contribute to the stimulated growth of HepG 2 cells both in vitro and in vivo. Considering that the HepG2 cell line was originated histologically from human hepatocellular carcinoma, our studies suggest that $\mathrm{HBx}$ can promote the progression of $\mathrm{HCC}$ via the activated Notch pathway.

In summary, our results demonstrate that $\mathrm{HBx}$ can act as an oncogenic factor to promote the progression of HCC by binding to NICD to activate the Notch signaling pathway, which may provide a new clue for the potential role of Notch signaling in the HBx-associated human liver carcinoma.

\section{Acknowledgements}

This study was supported by the National Science Foundation of China, no. 30570821 and no. 30971352. 


\section{References}

1. El-Serag HB: Hepatocellular carcinoma: recent trends in the United States. Gastroenterology 127: S27-S34, 2004.

2. Llovet JM, Burroughs A and Bruix J: Hepatocellular carcinoma. Lancet 362: 1907-1917, 2003.

3. Shibuya K, Mathers CD, Boschi-Pinto C, Lopez AD and Murray CJ: Global and regional estimates of cancer mortality and incidence by site: II. Results for the global burden of disease 2000. BMC Cancer 2: 37, 2002.

4. Koike K: Hepatitis B virus HBx gene and hepatocarcinogenesis. Intervirology 38: 134-142, 1995.

5. Lucito R and Schneider RJ: Hepatitis B virus X protein activates transcription factor NF-kappa B without a requirement for protein kinase C. J Virol 66: 983-991, 1992.

6. Kekule AS, Lauer U, Weiss L, Luber B and Hofschneider PH: Hepatitis B virus transactivator $\mathrm{HBx}$ uses a tumour promoter signalling pathway. Nature 361: 742-745, 1993.

7. Maguire HF, Hoeffler JP and Siddiqui A: HBV X protein alters the DNA binding specificity of CREB and ATF-2 by proteinprotein interactions. Science 252: 842-844, 1991.

8. Doria M, Klein N, Lucito R and Schneider RJ: The hepatitis B virus HBx protein is a dual specificity cytoplasmic activator of Ras and nuclear activator of transcription factors. EMBO J 14: 4747-4757, 1995.

9. Klein NP and Schneider RJ: Activation of Src family kinases by hepatitis B virus HBx protein and coupled signaling to Ras. Mol Cell Biol 17: 6427-6436, 1997.

10. Gottlob K, Fulco M, Levrero $M$ and Graessmann A: The hepatitis B virus HBx protein inhibits caspase 3 activity. J Biol Chem 273: 33347-33353, 1998.

11. Benn J and Schneider RJ: Hepatitis B virus HBx protein deregulates cell cycle checkpoint controls. Proc Natl Acad Sci USA 92: 11215-11219, 1995.

12. Ding Q, Xia W, Liu JC, et al: Erk associates with and primes GSK-3beta for its inactivation resulting in upregulation of betacatenin. Mol Cell 19: 159-170, 2005.

13. Benn J and Schneider RJ: Hepatitis B virus HBx protein activates Ras-GTP complex formation and establishes a Ras, Raf, MAP kinase signaling cascade. Proc Natl Acad Sci USA 91: 10350-10354, 1994

14. Lee YI, Kang-Park S and Do SI: The hepatitis B virus-X protein activates a phosphatidylinositol 3-kinase-dependent survival signaling cascade. J Biol Chem 276: 16969-16977, 2001.

15. Artavanis-Tsakonas S, Rand MD and Lake RJ: Notch signaling: cell fate control and signal integration in development. Science 284: 770-776, 1999.
16. Miele L: Notch signaling. Clin Cancer Res 12: 1074-1079, 2006.

17. Kopan R and Ilagan MX: Gamma-secretase: proteasome of the membrane? Nat Rev Mol Cell Biol 5: 499-504, 2004.

18. Maillard I, Adler SH and Pear WS: Notch and the immune system. Immunity 19: 781-791, 2003.

19. Jarriault S, Brou C, Logeat F, Schroeter EH, Kopan R and Israel A: Signalling downstream of activated mammalian Notch. Nature 377: 355-358, 1995.

20. Ohtsuka T, Sakamoto M, Guillemot F and Kageyama R: Roles of the basic helix-loop-helix genes Hes-1 and Hes5 in expansion of neural stem cells of the developing brain. J Biol Chem 276: 30467-30474, 2001.

21. Miele L, Golde T and Osborne B: Notch signaling in cancer. Curr Mol Med 6: 905-918, 2006.

22. Yanagawa S, Lee JS, Kakimi K, Matsuda Y, Honjo T and Ishimoto A: Identification of Notch-1 as a frequent target for provirus insertional mutagenesis in T-cell lymphomas induced by leukemogenic mutants of mouse mammary tumor virus. J Virol 74: 9786-9791, 2000.

23. Ellisen LW, Bird J, West DC, et al: TAN-1, the human homolog of the Drosophila notch gene, is broken by chromosomal translocations in T lymphoblastic neoplasms. Cell 66: 649-661, 1991.

24. Sjolund J, Manetopoulos C, Stockhausen MT and Axelson H: The Notch pathway in cancer: differentiation gone awry. Eur J Cancer 41: 2620-2629, 2005.

25. Nijjar SS, Crosby HA, Wallace L, Hubscher SG and Strain AJ: Notch receptor expression in adult human liver: a possible role in bile duct formation and hepatic neovascularization. Hepatology 34: 1184-1192, 2001

26. Nijjar SS, Wallace L, Crosby HA, Hubscher SG and Strain AJ: Altered Notch ligand expression in human liver disease: further evidence for a role of the Notch signaling pathway in hepatic neovascularization and biliary ductular defects. Am J Pathol 160: 1695-1703, 2002

27. Cheng B, Guo X, Zheng Y, Wang Y, Liu C and Li P: The effects of HBx gene on the expression of DNA repair enzymes hOGG1 and hMYHalpha mRNA in HepG2 cells. J Huazhong Univ Sci Technolog Med Sci 29: 187-192, 2009.

28. Gao J, Chen C, Hong L, et al: Expression of Jagged-1 and its association with hepatitis B virus X protein in hepatocellular carcinoma. Biochem Biophys Res Commun 356: 341-347, 2007.

29. Balint K, Xiao M, Pinnix CC, et al: Activation of Notch-1 signaling is required for beta-catenin-mediated human primary melanoma progression. J Clin Invest 115: 3166-3176, 2005. 